\title{
Nonmagnetic impurity in the spin-gap state
}

\author{
Naoto Nagaosa \\ Department of Applied Physics, University of Tokyo, Tokyo 113, Japan \\ Tai-Kai Ng \\ Department of Physics, HKUST, Clear Water Bay Road, Kowloon, Hong Kong \\ (Received 11 October 1994)
}

\begin{abstract}
The effects of nonmagnetic strong scatterers (unitary limit) on magnetic and transport properties are studied for resonating-valence-bond states in both the slave-boson and slave-fermion mean-field theories with the gap for the triplet excitations. In the $d$-wave pairing state of the slave-boson mean-field theory in two dimensions, there is no true gap for spinons, but the Anderson localization occurs, which leads to the local moment when the repulsive interaction is taken into account. In the slave-fermion mean-field theory, local moments are found bound to nonmagnetic impurities as a result of (staggered) gauge interaction. However, in both theories, localization of spinon does not appear in the resistivity, which shows the classical value for the holon.
\end{abstract}

Substitutions in high- $T_{c}$ cuprates offer an important means to study the strongly correlated metallic state of these compounds. Especially the effects of the nonmagnetic impurities, i.e., $\mathrm{Zn}$, replacing $\mathrm{Cu}$ in the conducting planes show unexpected features in the underdoped region. ${ }^{1-10}$

The formation of the magnetic moments due to $\mathrm{Zn}$ has been revealed by magnetic susceptibility, 1,8 NMR, ${ }^{2-5}$ $\mu \mathrm{SR},{ }^{6}$ and electron-paramagnetic resonance. ${ }^{7}$ Recent detailed study has shown that the moment is sitting on the nearest-neighbor $\mathrm{Cu}$ orbitals. ${ }^{5}$ The magnetic susceptibility shows Curie law and the Curie constant is roughly proportional to the $\mathrm{Zn}$ concentration and an almost full moment appears in the underdoped region, i.e., $0.8 \mu_{B}$ in $\mathrm{La}_{1.85} \mathrm{Sr}_{0.15} \mathrm{Cu}_{1-z} \mathrm{Zn}_{z} \mathrm{O}_{4}$ (Ref. 8) and $0.86 \mu_{B}$ in $\mathrm{YBa}_{2}\left(\mathrm{Cu}_{1-z} \mathrm{Zn}_{z}\right)_{3} \mathrm{O}_{6.64}$ (Ref. 5) per $\mathrm{Zn}$ ion. The magnitude of the induced local moments is strongly dependent on the hole concentration $x$ and becomes smaller or even vanishes as $x$ increases. 5,8

At an early stage Finkelstein et al. ${ }^{7}$ discussed the mechanism of this local moment formation, and put restrictions on the microscopic theories. The magnetic properties near the vacant site have been studied for the antiferromagnetically ordered state ${ }^{11}$ and for the cluster of $t-J$ model, ${ }^{12}$ but the local moments were not found.

Another important aspect of the impurity effects is the residual resistivity. $1,7,9,10$ It should be noted that the residual resistivity $\rho_{\text {res }}$ in the Boltzmann transport theory is independent of the effective mass of the carriers and is determined only by the phase shift $\delta_{0}$, the impurity concentration $n_{\text {imp }}$, and the carrier density $n$, i.e., $\rho_{\text {res }}=4\left(\hbar / e^{2}\right)\left(n_{\text {imp }} / n\right) \sin ^{2} \delta_{0}{ }^{8}$ In the Fermi-liquid theory the carrier number is that of the conduction electrons $1-x$ ( $x$ : hole concentration). However, experiments in $\mathrm{La}_{2-x} \mathrm{Sr}_{x} \mathrm{Cu}_{1-x} \mathrm{Zn}_{\mathrm{O}_{4}}$ and $\mathrm{YBa}_{2}\left(\mathrm{Cu}_{1-z} \mathrm{Zn}_{z}\right)_{3} \mathrm{O}_{y}$ have revealed that $N \cong x$ and $\delta_{0} \cong \pi / 2$ in the underdoped region. ${ }^{8}$ For example for $x=0.15, \quad z=0.01$ in $\mathrm{La}_{2-x} \mathrm{Sr}_{x} \mathrm{Cu}_{1-z} \mathrm{Zn}_{z} \mathrm{O}_{4}$ the localization effect does not show up down to the superconducting transition temperature $T_{c}$, and the temperature-independent increase of the resistivity is regarded as the residual resistivity $\rho_{\text {res }}$.
The obtained value $\rho_{\text {res }} \cong 1300 \Omega$ should be compared with the unitary limit value $\rho_{\text {unitary }}=1150 \Omega$ for $n=x$ and $\rho_{\text {unitary }}=200 \Omega$ for $n=1-x$, and the carrier density $n$ should be identified with that of the doped hole $x .^{8}$ As the hole concentration $x$ increases with fixed $z$, the residual resistivity decreases and eventually $n$ is identified with $1-x$ as in the usual Fermi liquid.

In this paper we shall explain these two aspects, i.e., transport and magnetic properties of the underdoped samples, in a unified way from the standpoint of the resonating-valence-band theories. In the framework of the slave-boson mean-field theory, the local-spin moments are due to the Anderson localization of the spinons in the $d$-wave pairing state whereas in the slave-fermion mean-field theory, they appear because of the strong (staggered) gauge interaction which binds spinons to the nonmagnetic impurities. In both theories the resistivity is determined by the residual resistivity of the holons by the Boltzmann transport theory.

First let us consider the local moment induced by the nonmagnetic impurities. We have two examples in 1D where the local moment is induced by the nonmagnetic impurity. An example is the double-chain Heisenberg antiferromagnet, where the gap for the triplet excitations is opened. In this system a nonmagnetic impurity gives rise to a spin- $-\frac{1}{2}$. However the local-spin moment does not appear near the edge of the sample as will be described below. Another well-known example with the gap is the Haldane system with $S=1 . .^{13}$ If one introduces a nonmagnetic impurity and cuts the chain, the local spin- $\frac{1}{2}$ appears near the edge of the chain within the extent of the order of $J / E_{H}$ times the lattice constant ( $J$ exchange coupling, $E_{H}$ Haldane gap). Although these are onedimensional (1D) examples, we shall embody below the analogy between one and two dimensions.

We start with the purely spin model

$$
\begin{aligned}
H= & J \sum_{j=1, L} \sum_{i=-\infty, \infty} \mathbf{S}_{i j} \cdot \mathbf{S}_{i+1 j} \\
& +J^{\prime} \sum_{j=1, L-1} \sum_{i=-\infty, \infty} S_{i j} \cdot \mathbf{S}_{i j+1},
\end{aligned}
$$


where $J$ is the intrachain exchange and positive (antiferromagnetic) and $J^{\prime}$ is the interchain exchange coupling. We take the open boundary condition along the $y$ direction. $L=2$ and $J^{\prime} \rightarrow-\infty$ corresponds to the $S=1$ antiferromagnetic chain (Haldane system), while $L=2, J^{\prime}=J$ to the double-chain Heisenberg antiferromagnet. Let us start with the antiferromagnetic $J^{\prime}(>0)$. In this case the slave-boson theory ${ }^{14-16}$ is more convenient because the order parameter for the singlet formation can be more easily identified. In this scheme the spin operator $\mathbf{S}_{i j}$ with spin $\frac{1}{2}$ is expressed in terms of the fermion (spinon) operators as $\mathbf{S}_{i j}=(1 / 2) f_{i j \alpha}^{\dagger} \boldsymbol{\sigma}_{\alpha \beta} f_{i j \beta}$ with the constraint $\sum_{\alpha=\uparrow, \downarrow} f_{i j \alpha}^{\dagger} f_{i j \alpha}=1$ for each site. Using these fermion operators, the Hamiltonian Eq. (1) is expressed in terms of these fermion (spinon) operators, and we employ the mean-field theory with the order parameters $\chi_{x}(i j)=\left\langle\sum_{\alpha} f_{i j \alpha}^{\dagger} f_{i+1 j \alpha}\right\rangle$ and $\chi_{y}(i j)=\left\langle\Sigma_{\alpha} f_{i j \alpha}^{\dagger} f_{i j+1 \alpha}\right\rangle$. Then we obtain

$$
\begin{aligned}
H_{\mathrm{MP}}= & -J \sum_{i j} \sum_{\alpha} \chi_{x}(i j) \sum_{\beta} f_{i+1 j \beta}^{\dagger} f_{i j \beta}+\text { H.c. } \\
& -J^{\prime} \sum_{i j} \sum_{\alpha} \chi_{y}(i j) \sum_{\beta} f_{i j+1 \beta}^{\dagger} f_{i j \beta},+ \text { H.c. }
\end{aligned}
$$

We take here the $\pi$-flux state where each plaquette encloses $\pi$ flux, i.e., $\chi_{x}(i j)=\chi_{x}=$ const, $\chi_{y}(i j)=(-1)^{i} \chi_{y},{ }^{15}$ because it reproduces the qualitatively correct features in finite width $(L)$ systems. (One can choose another solution which is equivalent to that above by the gauge transformation $f_{i j \alpha} \rightarrow f_{i j \alpha} e^{i \theta_{i j}}$.) Then we obtain the two bands for the spinons whose dispersions are given by

$$
E_{ \pm}(\mathbf{k})= \pm \sqrt{\left(J \cos k_{x}\right)^{2}+\left(J^{\prime} \cos k_{y}\right)^{2}} \text {. }
$$

Because of the open boundary condition, $k_{y}$ is discretized as $k_{y}=\pi n_{y} /(L+1)\left(n_{y}\right.$ : integer) and it is easily seen that $k_{y}$ can take $\pi / 2$ and there is no gap for $L=$ odd, while there appears a gap for $L=$ even. This even-odd effect has been already discussed theoretically ${ }^{17}$ and confirmed experimentally. ${ }^{18}$ Both even and odd series converge to the $\pi$-flux state in 2D as $L \rightarrow \infty$. Now let us put one nonmagnetic impurity, i.e., an inert site where the fermion is excluded. It is convenient to express this situation by introducing the potential $V_{0}$ at the impurity site and take the limit $V_{0} \rightarrow \infty$ afterward. This is solved by the SlaterKoster method ${ }^{19}$ and the advanced Green's function $G(\omega)$ for the impurity site is obtained as $G(\omega)=G_{0}(\omega) /\left[1-V_{0} G_{0}(\omega)\right]$, where $G_{0}(\omega)$ is the Green's function in the absence of the impurity and is given by $G_{0}(\omega)=\int d E D(E) /(\omega-i \delta-E)$ with $D(E)$ being the density of the one-particle states per one lattice site. In the present case there is the particle-hole symmetry, and $D(E)=D(-E)$. The $L$ dependence of the density of state is as follows. For odd $L$ there is the finite density of states for $E=0$ but its weight is $\sim L^{-1}$. For even $L$ there opens a gap around $E=0$ which decreases as $\sim L^{-1}$. In the limit $L \rightarrow \infty$ both of these series converges to that of the 2D flux state where $D(E) \propto|E|$ for small $|E|$. If one takes the even $L$ series, $G_{0}(\omega)$ can be expanded around $\omega=0$ as $G_{0}(\omega)=-\alpha \omega+O\left(\omega^{2}\right)$ with $\alpha=\int d E D(E) / E^{2}$. Putting this $G_{0}$ into the expression for $G$, we obtain $G(\omega) \cong-\alpha \omega /\left(1+\alpha V_{0} \omega\right)$, which has a pole at $\omega=-1 / \alpha V_{0} \rightarrow 0$ as $V_{0} \rightarrow \infty$. One can calculate the change of the density of states $\Delta D(\omega)$ as

$$
\Delta D(\omega)=\frac{1}{\pi} \frac{\partial}{\partial \omega} \operatorname{Im} \ln \left[1-V_{0} G_{0}(\omega)\right]=\delta(\omega),
$$

which means the appearance of a localized state in the middle of the gap. The extent of this localized state is estimated to be of the order of (band width)/(gap) $\times$ (lattice constant) as in the case of impurity state in semiconductors. When one takes into account the repulsive interaction between the fermions which is neglected in the mean-field approach but actually exists, only one fermion can occupy this mid-gap as in the case of the spin soliton in polyacetylene which leads to the localized spin moment.

Now we consider the edge effect in this even-width spin system with a gap. We take the ladder $(L=2)$ case for simplicity, and put two nonmagnetic impurities on the neighboring sites along the $y$ axis which cut the ladder. The analysis above can be generalized to the present case by introducing $G(\omega)$ of $2 \times 2$ matrix, and it can be easily seen that there appear no localized states in the spin gap. This is because the antiferromagnetic $J^{\prime}$ along the $y$ axis make the singlet even near the edge, which is in contrast to the ferromagnetic $J^{\prime}$, i.e., Haldane system, which will be discussed shortly.

As $L$ increases, also the extent of the localized state increases and finally the interference of the scattered wave from the different scatterers becomes important for finite concentration of the impurities. This is exactly the problem of the Anderson localization ${ }^{20}$ and we now study it in the two-dimensional limit. Actually the same problem has been already studied by Lee ${ }^{21}$ in the context of the localized states in $d$-wave superconductors. As has been discussed by Affleck et al. ${ }^{16}$ there is the $S U(2)$ symmetry corresponding to the particle-hole transformation in the fermion representation, and the $\pi$-flux state is equivalent to the $d$-wave pairing state for the spin model. (When the holes are doped this SU(2) symmetry disappears, which will be discussed later.) Therefore we can apply his results to the $\pi$-flux state with a slight modification. The strong scatterer considered in this paper corresponds to his model II. ${ }^{21}$ In this model the self-energy $\Sigma(\omega)$ is determined self-consistently by $\Sigma(\omega)=-\Gamma / g_{0}(\omega)$ where $\Gamma=n_{\text {imp }} /\left(\pi \rho_{0}\right), g_{0}(\omega)=4 /\left(\pi \rho_{0}\right) \Sigma_{\mathbf{k}} G(\mathbf{k}, \omega) . \quad\left(n_{\text {imp }}\right.$ is the density of the nonmagnetic impurities per area, and $\rho_{0}$ is the density of states in the normal state in the absence of the $d$-wave pairing.) The imaginary part $\gamma_{0}$ of the selfenergy near the Fermi level is obtained by solving $\gamma_{0}=-\Gamma / g_{0}\left(\gamma_{0}\right)$. In the scaling theory of localization, the localization length $l_{\text {loc }}$ is estimated to be $l_{\text {loc }} \sim l \exp \left(k_{F} l\right)$ with $l$ being the elastic mean free path. $l$ is estimated from the classical conductance and hence the imaginary part of the self-energy $\gamma(\omega)$, which behaves as $\gamma_{0} \cong \gamma_{0}$ for $|\omega|<\gamma_{0}$, while $\gamma(\omega) \cong \gamma_{0}^{2} / \omega$ for $|\omega|>\gamma_{0}$. Therefore it can be considered that the states within the region $|\omega|<\gamma_{0}$ are strongly localized. The density of these strongly localized states $n_{\text {loc }}$ per area is estimated as the density of states for this energy region $-\pi \rho_{0} g_{0}\left(\gamma_{0}\right)$ times the energy interval $\gamma_{0}$, and we obtain $n_{\text {loc }} \cong-\pi \gamma_{0} \rho_{0} g_{0}\left(\gamma_{0}\right)=\pi \rho_{0} \Gamma=n_{\text {imp }}$. Now we have shown that the number of strongly localized states roughly coin- 
cides with the number of nonmagnetic impurities. When the repulsive interaction between the fermions are again taken into account, it is expected that only these states near the Fermi level are singly occupied and give rise to the Curie moments proportional to the impurity density.

In the slave-fermion mean-field theory (SFMFT) for Heisenberg antiferromagnets ${ }^{22}$ with nonmagnetic impurities, the fermion spin operators $f_{i j \sigma}$ are replaced by bosonic spin operators $Z_{i j \sigma}$ with constraint $\sum_{\sigma} \bar{Z}_{i j \sigma} Z_{i j \sigma}=2 S\left(1-n_{i j}^{I}\right)$ for each site $(i, j)$, where $n_{i j}^{I}=1$ if site $(i, j)$ is occupied by a nonmagnetic impurity and is zero otherwise. $S$ is the spin magnitude. The Hamiltonian is then decoupled in the Cooper channel by introducing order parameters

$$
\Delta_{i j}^{(x)}=\left\langle Z_{i j \uparrow} Z_{i+1 j \downarrow}-Z_{i j \downarrow} Z_{i+1 j \uparrow}\right\rangle
$$

and

$$
\Delta_{i j}^{(y)}=\left\langle Z_{i j \uparrow} Z_{i j+1 \downarrow}-Z_{i j \downarrow} Z_{i j+1 \uparrow}\right\rangle .
$$

The mean-field Hamiltonian is

$$
\begin{aligned}
H_{\mathrm{MF}}= & -J \sum_{\langle i j\rangle} \Delta_{i j}^{(x) *}\left(Z_{i j \uparrow} Z_{i+1 j \downarrow}-Z_{i j \downarrow} Z_{i+1 j \uparrow}\right)+\text { H.c. } \\
& -J^{\prime} \sum_{\langle i j\rangle} \Delta_{i j}^{(y) *}\left(Z_{i j \uparrow} Z_{i j+1 \downarrow}-Z_{i j \downarrow} Z_{i j+1 \uparrow}\right)+\text { H.c . } \\
& +\sum_{i j \sigma} \lambda_{i j}\left[\bar{Z}_{i j \sigma} Z_{i j \sigma}+2 S\left(n_{i j}^{I}-1\right)\right] .
\end{aligned}
$$

In the pure case when nonmagnetic impurities are absent, $\Delta$ 's and $\lambda$ 's are independent of position and $H_{\mathrm{MF}}$ can be diagonalized easily. We obtain dispersion for the (bosonic) spinon eigenstates $\gamma_{\mathbf{k} \sigma}$ with

$$
H_{\mathrm{MF}}=\sum_{\mathbf{k} \sigma} E_{\mathbf{k}} \gamma_{\mathbf{k} \sigma}^{+} \gamma_{\mathbf{k} \sigma}+E_{G}
$$

where

$$
E_{\mathbf{k}}=\sqrt{\lambda^{2}-\left(J \Delta^{(x)} \cos k_{x}+J^{\prime} \Delta^{(y)} \cos k_{y}\right)^{2}}
$$

and $E_{G}$ is the ground-state energy. Notice that spin-gap phases are obtained if $\lambda^{2}>\left(J \Delta^{(x)}+J^{\prime} \Delta^{(y)}\right)^{2}$. Such a spingap phase exists for all value of $S$ in one dimension in SFMFT. This SFMFT together with the gauge field shortly introduced give an adequate description of $S=$ integer spin chains, while it fails to describe the massless state of $S=$ half-integer spin chains. This is because the topological Berry phase term which plays a crucial role in the latter case is not taken into account correctly in SFMFT. Therefore the following arguments can be applied only to the $S=$ integer case in $1 \mathrm{D}$. In $2 \mathrm{D}$, the topological term is canceled between neighboring chains in the effective Lagrangian and SFMFT is more reliable. In $2 \mathrm{D}$ and in the isotropic case $J=J^{\prime}$, spin-gap phase exists in SFMFT only when $S<S_{c}$, where $S_{c} \sim 0.19 .^{22}$ Nevertheless, in the problem of high- $T_{c}$ superconductors considered here, we shall assume that the normal-state magnetic properties in the underdoped region can be described by the spin-gap phase of SFMFT and shall examine the effect of nonmagnetic impurities under this assumption.

In the spin-gap phase, it has been shown that the lowenergy dynamics of the spin system can be described by an effective Lagrangian of charged $S=\frac{1}{2}$ bosons (spinons) coupled to $U(1)$ gauge field, ${ }^{23}$

$$
\begin{aligned}
L_{\mathrm{eff}}= & \left|\left(\partial_{\mu}-i A_{\mu}\right) Z\right|^{2}+m^{2}|Z|^{2}+\frac{1}{e^{2}} F_{\mu \nu}^{2} \\
& +2 S A_{0} e_{i j} n_{i j}^{I}
\end{aligned}
$$

where $Z^{\dagger}=\left(Z_{\uparrow}^{*}, Z_{\downarrow}^{*}\right)$ is a two-component $S=\frac{1}{2}$ spinor field carrying unit gauge charge, $F_{\mu \nu}=\partial_{\mu} A_{\nu}-\partial_{\nu} A_{\mu}$ is the usual $U(1)$ gauge field, and $A_{0}$ is the scalar potential in the Coulomb gauge. Moreover, it can be shown that $e^{2} \sim m^{2}$ in $1 \mathrm{D}$ and $e^{2} \sim m$ in $2 \mathrm{D}$.

It is important to distinguish between the "uniform" and "staggered" gauge fields in the slave-fermion approach. In SFMFT, the bosons (spinons) are naturally divided into two classes according to their sublattices because of the underlying antiferromagnetic correlation. The "uniform" gauge field is a phase field which couples to bosons on both sublattices with same gauge charge, whereas the "staggered" gauge field couples to them with opposite gauge charge. ${ }^{23}$ In terms of the phase $\theta_{j}^{(\alpha)}$ (where $\alpha=x, y$ ) of the order parameter $\Delta_{i j}^{(\alpha)}$ $=D_{i j}^{(\alpha)} \exp \left(i \theta_{i j}^{(\alpha)}\right),(D=|\Delta|)$, the uniform and staggered gauge fields $A^{(\alpha)}(\mathbf{k})$ and $M^{(\alpha)}(\mathbf{k})$ in Fourier space are equal to $(1 / 2)\left[\theta^{(\alpha)}(\mathbf{k})+(-) \theta^{(-\alpha)}(\mathbf{k})\right]$, respectively, whereas $M^{(0)}(\mathbf{k})$ are given by $1 / s\left[\lambda^{A}(\mathbf{k})+(-) \lambda^{B}(\mathbf{k})\right]$, where $A, B$ are sublattice indices. In SFMFT, the uniform gauge symmetry is broken because of the existence of nonzero order parameter $\Delta$, whereas the "staggered" gauge symmetry remains unbroken. The low-energy dynamics of the system is dominated by the staggered gauge field, described by the effective Lagrangian, Eq. (7). ${ }^{23}$ For this reason $e_{i j}=+(-) 1$ for impurities living on $A(B)$ sublattices.

In $1 \mathrm{D}$, introduction of a single nonmagnetic impurity in a quantum spin chain effectively breaks the spin chain into two disjoint pieces. For integer $S$ spin chains where spin gap exists, it is known that $S^{\prime}=S / 2$ edge states are being generated at the edge of the broken chain. ${ }^{24}$ The existence of edge states around a nonmagnetic impurity can be explained using the effective Lagrangian in SFMFT approach. Notice that in $L_{\text {eff }}$, impurities appear as external (staggered) gauge charges of magnitude $2 S$ localized at the impurity sites. In $1 \mathrm{D}, U(1)$ gauge field is confining and it is always energetically favorable to nucleate charged bosons (spinons) from vacuum to screen the electric field. For integer spin chains, $S$ bosons are nucleated at each side of the impurity site forming $S=S / 2$ edge states at each side of the impurity site ( recall that each boson carries spin $1 / 2$ ). In $2 \mathrm{D}$, a similar phenomenon occurs except that the corresponding electrostatic potential $V(r) \sim e^{2} \ln (r / \xi) \quad\left(\xi \sim m^{-1}\right.$ is the coherence length) is much weaker than the linear confining potential in 1D. Nevertheless $2 S$ bosons or local moment of magnitude $S$ is still expected to be confined around a nonmagnetic impurity giving rise to Curie-Weiss behavior in spin susceptibility.

For finite concentration of impurities the discussion has to be more careful. Notice that a finite amount of energy $\sim m$ is needed to nucleate one boson. Thus for finite concentration of impurities $\delta$, the total energy of the sys- 
tem has to be considered to see whether it is energetically favorable to bind bosons to impurities. The electrostatic energy gained by nucleating bosons to screen the impurities is of order $(2 S e)^{2} \delta\left[\ln (1 / \xi)-\ln \left(l_{0} / \xi\right)\right]$ where $l \sim \delta^{-1 / 2}$ is the average distance between nonmagnetic impurities and $l_{0} \sim \xi$ is the typical size of a spinon. Thus the total energy required to nucleate bosons to screen the impurities is of order $2 S \delta\left[m-2 S e^{2} \ln (l / \xi)\right]$. Notice that in $2 \mathrm{D}, e^{2} \sim m$. Thus for $S=\frac{1}{2}$ the two terms are of comparable magnitude when $l \sim \xi$, implying that local moments are formed only when the concentration of nonmagnetic impurities is small, and will be quenched when density of impurities is large enough, in general agreement with experimental observation.

We now turn to the doped case. In the slave-boson approach the electron operators are expressed in terms of those of spinons $f^{\dagger}, f$ and holons $b^{\dagger}, b$ as ${ }^{25}$

$$
C_{i j \alpha}=f_{i j \alpha} b_{i j}^{\dagger} \text {. }
$$

In this case the $S U(2)$ symmetry discussed above is broken and we have to distinguish between the bond order $\chi=\Sigma_{\alpha}\left\langle f_{\alpha}^{\dagger} f_{\alpha}\right\rangle$ and the singlet pairing $\Delta=\left\langle f_{\uparrow} f_{\downarrow}\right.$ $\left.-f_{\downarrow} f_{\uparrow}\right\rangle$. Including also the bond order $\chi_{h}$ and Bose condensation $B$ of the holons, the mean-field phase diagram has been discussed. In the spin-gap phase which we are now interested in, the bond order parameters $\chi, \chi_{h}$ as well as the spinon pairing $\Delta$ is nonzero, while the holon condensation does not occur $(B=0)$. In this phase, the spinon is superconducting, and the physically observable resistivity is determined by that of the holons thermally distributed near the zero momentum. The effects of the nonmagnetic impurities are essentially unaltered from those discussed above, but the localization does not suppress completely the spinon pairing, i.e., the quasiparticles are localized and the spinon conductivity remains infinite. Even when the gauge field is taken into account and the superconductivity of the spinons is in the fluctuating phase, the conductivity of spinons is expected to be still very large. Thus the localized moment and the metallic transport can coexist when the spin-charge separation occurs. As for the holons, the density of states

${ }^{1}$ G. Xiao, M. Z. Cieplak, J. Q. Xiao, and C. L. Chien, Phys. Rev. B 35, 8782 (1987).

${ }^{2}$ H. Alloul et al., Phys. Rev. Lett. 67, 3140 (1991).

${ }^{3}$ G. Zheng et al., J. Phys. Soc. Jpn. 62, 2591 (1993).

${ }^{4}$ K. Ishida et al., J. Phys. Soc. Jpn. 62, 2803 (1993).

${ }^{5}$ A. V. Mahajan, H. Alloul, G. Collins, and J. F. Marucco, Phys. Rev. Lett. 72, 3100 (1994).

${ }^{6}$ P. Mendels et al., Phys. Rev. B 49, 10035 (1994).

${ }^{7}$ A. M. Finkelstein, V. E. Kataev, E. F. Kukovitskii, and G. B. Teitel'baum, Physica C 168, 370 (1990).

${ }^{8} \mathrm{~S}$. Uchida (unpublished).

${ }^{9}$ S. Ikegawa et al., Phys. Rev. B 43, 2885 (1991).

${ }^{10}$ K. Semba, A. Matsuda, and T. Ishii, Phys. Rev. B 49, 10043 (1994).

${ }^{11}$ N. Nagaosa, Y. Hatsugai, and M. Imada, J. Phys. Soc. Jpn. 58, 9781 (1989).

${ }^{12}$ D. Poiblanc, D. J. Scalapino, and W. Hanke, Phys. Rev. Lett. 72, 884 (1994).

${ }^{13}$ M. Hagiwara et al., Phys. Rev. Lett. 65, 3181 (1990). near the bottom of the band is not small in 2D, and the criterion of the localization is the resistivity observed in experiments. It is also noted that the strong inelastic scattering suppresses the localization effect down to $T_{c}$, and the residual resistivity can be estimated by the classical Boltzmann transport theory. Because the carriers are the holons and not the spinons, the carrier number $n$ appearing in the expression for $\rho_{\text {res }}$ should be $x$ in agreement with experiment.

In the slave-fermion approach the electron operators are expressed in a similar way as in Eq. (8), except that the statistics are inverted, i.e., $C_{i j \alpha}=Z_{i j \alpha} f_{i j}^{\dagger}$, where now $Z$ 's are Schwinger bosons and $f$ 's are slave fermions. In the $t^{\prime}-J$ model where spiral phases do not occur, the mean-field theory describes gases of bosonic spinons and fermionic holons interacting through gauge fluctuations. ${ }^{26}$ In the spin-gap phase, the spinons are superconducting with respect to the uniform gauge field. Thus the physically observable resistivity is determined by that of the holons which describe a spinless Fermi gas with density of holes $\delta$, giving rise naturally to $\rho_{\text {res }}$ of form discussed in the introduction.

In summary we have studied the effect of the nonmagnetic impurities on the magnetic and transport properties in the spin-gap state. In the slave-boson approach, the induced local-spin moment is explained by the localization effect together with the repulsive interaction whereas in the slave-fermion approach, local-spin moment appears as a result of staggered gauge interaction. It is noted that the number of localized moments are roughly that of the impurities in the unitary scattering limit. This localization effect of magnetic moment, however, does not appear in the resistivity, which is determined by the holons and the carrier number $n$ is the hole number $x$ in contrast to the Fermi-liquid case where $n=1-x$.

One of the authors (N.N.) acknowledges Professor S. Uchida, Professor Y. Kitaoka, Professor H. Takagi, and Dr. M. Sigrist for fruitful discussions. This work was supported by Grant-in-Aid for Scientific Research No. 05044037, No. 04240103, and No. 04231105 from the Ministry of Education, Science, and Culture of Japan.

${ }^{14}$ G. Baskaran, Z. Zou, and P. W. Anderson, Solid State Commun. 63, 1233 (1991).

${ }^{15}$ I. Affleck and J. B. Marston, Phys. Rev. B 37, 3774 (1988).

${ }^{16}$ I. Affleck, Z. Zou, T. Su, and P. W. Anderson, Phys. Rev. B 38, 745 (1988).

${ }^{17}$ T. M. Rice, S. Gopalan, and M. Sigrist, Europhys. Lett. 23, 445 (1993).

${ }^{18} \mathrm{~K}$. Ishida et al., J. Phys. Soc. Jpn. 63, 3222 (1994).

${ }^{19}$ See, for e.g., G. Mahan, Many-Particle Physics (Plenum, New York, 1981), Chap. 4.

${ }^{20}$ P. A. Lee and T. V. Ramakrishnan, Rev. Mod. Phys. 57, 287 (1985).

${ }^{21}$ P. A. Lee, Phys. Rev. Lett. 71, 1887 (1993).

${ }^{22}$ D. P. Arovas and A. Auerbach, Phys. Rev. B 38, 316 (1988).

${ }^{23}$ N. Read and S. Sachdev, Phys. Rev. Lett. 62, 1694 (1989).

${ }^{24}$ T. K. Ng, Phys. Rev. B 50, 555 (1994).

${ }^{25}$ P. A. Lee and N. Nagaosa, Phys. Rev. B 46, 5621 (1992).

${ }^{26}$ X. G. Wen, Phys. Rev. B 39, 7223 (1989). 